\title{
A bioassay for studying behavioural responses of the common bed bug, Cimex lectularius (Hemiptera: Cimicidae) to bed bug-derived volatiles
}

\author{
E.N.I. Weeks ${ }^{1,2 *}$, J.G. $\operatorname{Logan}^{1}$, S.A. Gezan ${ }^{1}$, \\ C.M. Woodcock ${ }^{1}$, M.A. Birkett ${ }^{1}$, J.A. Pickett ${ }^{1}$ \\ and M.M. Cameron ${ }^{2}$
}

${ }^{1}$ Centre for Sustainable Pest and Disease Management, Biological Chemistry Department, Rothamsted Research, Harpenden, Hertfordshire, UK, AL5 2JQ: ${ }^{2}$ Disease Control and Vector Biology Unit, Department of Infectious Tropical Diseases, London School of Hygiene and Tropical Medicine, London, UK, WC1E 7HT

\begin{abstract}
The common bed bug, Cimex lectularius (Hemiptera: Cimicidae), has recently re-emerged in increasing numbers, distribution and intensity of infestation in many countries. Current control relies on the application of residual pesticides; but, due to the development of insecticide resistance, there is a need for new tools and techniques. Semiochemicals (behaviour and physiology modifying chemicals) could be exploited for management of bed bugs. However, in order to identify semiochemicals that can be utilised in monitoring or control, a suitable olfactometer is needed that enables the study of the responses of bed bugs to volatile chemicals. Previous studies have used olfactometers that do not separate olfactory responses from responses to physical contact. In this study, a still-air olfactometer was used to measure behavioural responses to different bed bug-derived volatiles presented in an odour pot. Bed bugs were significantly more likely to visit the area above the odour pot first, and more frequently, in the presence of volatiles from bed bug-exposed paper but not in the presence of volatiles from conspecific bed bugs. Bed bug activity was found to be dependent on the presence of the volatiles from bed bug-exposed paper, the time during the scotophase and the sex of the insect being tested. The still-air olfactometer could be used to test putative semiochemicals, which would allow an understanding of their behavioural role in bed bug ecology. Ultimately, this could lead to the identification of new semiochemical tools for bed bug monitoring and control.
\end{abstract}

Keywords: Cimex lectularius, aggregation, olfactometer, behaviour, semiochemical

(Accepted 4 October 2009)

*Author for correspondence

Fax: + 441582762595

E-mail: emma.weeks@bbsrc.ac.uk 


\section{Introduction}

The common bed bug, Cimex lectularius (Hemiptera: Cimicidae), which feeds nocturnally upon the blood of human hosts, is an important medical and veterinary pest in the temperate world (Boase, 2004). During the past decade, this once common pest has re-emerged in increasing numbers, distribution and intensity of infestation in the US, Canada, Europe, Australia and Africa (Harlan, 2006a,b; Romero et al., 2007). For example, there was an increase in calls made by the public to pest control operators of $300 \%$ in Atlanta, USA from 2000-2001, a 400\% increase in complaints in Australia from 2001-2004, and an average increase of calls concerning bed bugs, as opposed to other household pests, of 24.7 calls per year from 2000-2006 in Greater London (Doggett et al., 2004; Harlan et al., 2008, Richards et al., 2009).

The only reliable monitoring method currently available involves the search for evidence of an active infestation, which is labour intensive and time consuming. Sticky traps are occasionally used but are not effective for low-level infestations (Potter, 2005; Harlan, 2007; Harlan et al., 2008). Bed bug control is currently reliant on residual insecticides, usually pyrethroids or carbamates, but several recent studies have shown the development of pyrethroid resistance in Cimicidae due to both indoor residual spraying in developed countries (Romero et al., 2007; Harlan et al., 2008; Moore \& Miller, 2009) and the use of insecticide-treated bed nets in developing countries (Myamba et al., 2002; Weerakone, 2007). Additionally, the withdrawal of organophosphates and carbamates from the UK and US markets have reduced the number of available effective insecticides (Harlan et al., 2008; Potter et al., 2008). Therefore, there is a need for new approaches for bed bug monitoring and control, for example, the addition of semiochemicals, such as aggregation pheromones, to currently available traps, changing them from simple interceptive devices, such as sticky traps, to species-specific odour-baited traps. These traps would intercept refuge-seeking bugs by attracting them into the device through behavioural manipulation with semiochemicals.

For the development of such trapping systems, identification of semiochemicals and further information about the behavioural roles of the identified compounds is necessary. Identification of the key semiochemicals will enable the production of economical odour baits. In addition, it is important to understand fully the role of the chemical in the insects' biology and behaviour before manipulation is attempted. Different semiochemicals will be perceived by bed bugs with varying effects on behaviour, which could influence the design of the trap and its ultimate efficacy as a monitoring device. It is also vital, for monitoring of this species, that a trap should catch every life stage, as all stages are haematophagous and cause potential harm. To identify semiochemicals that could be used to control bed bugs, an appropriate method for assessing behavioural responses is essential. Olfactometers have been investigated in the past, but they do not always take into account the natural crevice-dwelling behaviour of bed bugs, which are positively thigmotaxic, i.e. prefer to maintain close contact with conspecifics and surfaces within their refuges (Aboul-Nasr \& Erakey, 1968a) and are not usually exposed to a strong air flow (Siljander et al., 2007). Indeed, bed bugs tend to avoid air flow where possible. When subjected to moving air, for example in a Petterson four-way olfactometer, bed bugs turned away, lowered their antenna and retracted their legs (Aboul-Nasr \& Erakey, 1968a). Previous studies that have successfully tested the response of bed bugs to chemical stimuli have done so in still-air olfactometers (Aboul-Nasr \& Erakey, 1968b; Levinson \& Bar Ilan, 1971; Levinson et al., 1974b; Parashar et al., 2003; Siljander et al., 2007, 2008). Some of these studies used groups of 10-30 bed bugs (Levinson \& Bar Ilan, 1971; Levinson et al., 1974b; Parashar et al., 2003), but testing groups of bed bugs can lead to confusing results due to the interactions between individuals, as they are known to aggregate and produce an alarm pheromone (Aboul-Nasr \& Erakey, 1968a; Levinson et al., 1974b; Marx, 1955). More recent studies introduced individual insects into a still-air olfactometer (Siljander et al., 2007, 2008). However, as all of these studies allow bed bugs to rest on treated papers, they do not discriminate between odour responses (chemotaxis) and responses due to physical contact (thigmotaxis) (Levinson \& Bar Ilan, 1971; Levinson et al., 1974a; Parashar et al., 2003; Siljander et al., 2007, 2008; Olson et al., 2009). When contact stimuli are removed, attraction to the odour is usually lost (Siljander et al., 2007). It is important that olfactometers allow the separation of chemotaxis from thigmotaxis, in order to determine the true effect of the volatile chemicals on bed bug behaviour. Additionally, olfactometers previously used were all final choice bioassays, and the intermediate behaviour was not taken into consideration. Studying behavioural responses over a short time frame provides a greater understanding of the behavioural role of the volatiles, which will become much more relevant when moving from laboratory to field testing. In addition, it is the first, not the final, choice that is important for the success of the trap as a monitoring device, as the bed bugs will be caught on first contact.

An alternative bioassay is the still-air Petri-dish olfactometer, comprising a two-choice arena in which one half differs from the other by only one variable at a time. In this case, the difference between the two halves of the arena is the presence or absence of the volatile chemical/s. The olfactometer prevents direct contact of the insect with the volatile source and has been used to measure the response of the vetch aphid, Megoura viciae, to a sex pheromone (Dawson et al., 1987). In the still-air Petri-dish olfactometer, bed bug behaviour can be studied in an environment with limited air flow. The position of the bed bug can be recorded along with activity variables, allowing an understanding of the behavioural response of the bed bug to the volatiles over a short time period. In addition, the olfactometer prevents contact of the bed bug with the volatile source, facilitating the separation of chemotactic and thigmotactic responses.

The aim of this study was to assess the suitability of the still-air Petri-dish olfactometer for observing bed bug behaviour. Behaviour was observed in response to bed bug-derived volatiles that would be found naturally occurring in bed bug refuges.

\section{Materials and methods}

Insects

Cimex lectularius were donated from a laboratory culture maintained at the London School of Hygiene and Tropical Medicine and then maintained at Rothamsted Research. The 


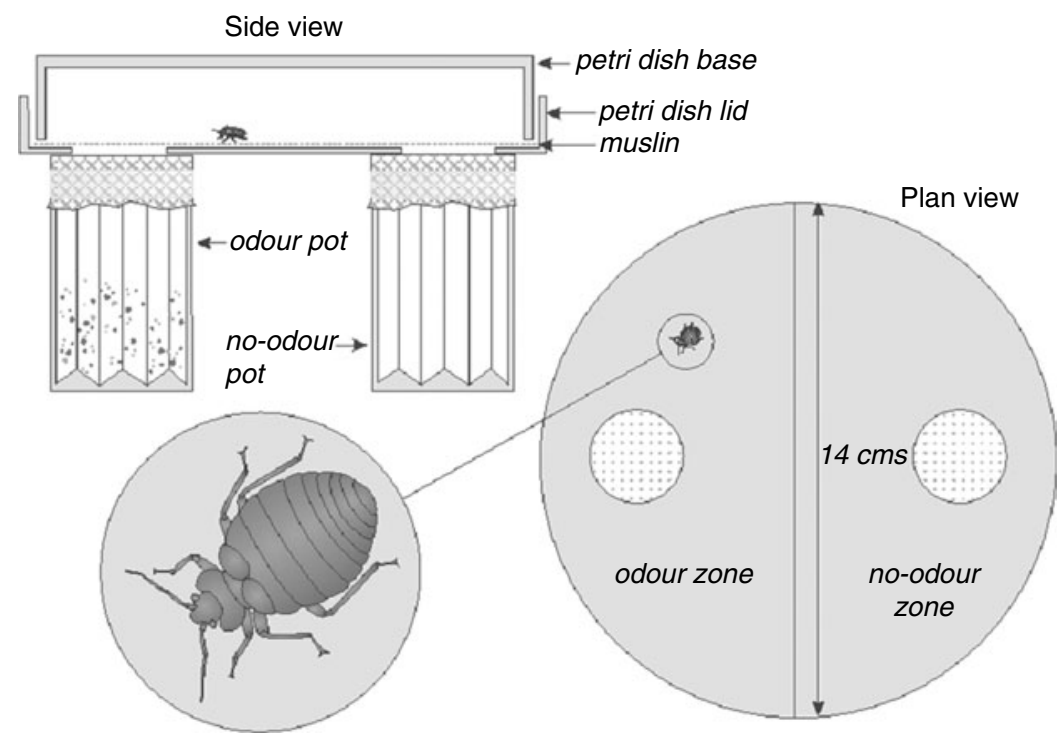

Fig. 1. Illustration of the still-air olfactometer showing side and plan view. Internal diameter $140 \mathrm{~mm}$, hole diameter $26 \mathrm{~mm}$, distance between holes $64 \mathrm{~mm}$. Pots were plastic and $60 \times 40 \mathrm{~mm}$. Odour pot containing filter paper with bed bug-derived volatiles. No-odour pot containing clean filter paper.

bed bugs were reared in plastic colony pots $(60 \times 40 \mathrm{~mm})$ in a controlled environment at $25^{\circ} \mathrm{C} \pm 1.5^{\circ} \mathrm{C}$ and $80 \% \mathrm{RH} \pm 5 \%$. The light regime was set to $14 \mathrm{~L}: 10 \mathrm{D}$. Bed bugs were given access to heparinised sheep blood (TCS Biosciences, UK) once per week via an artificial blood-feeding system (Montes et al., 2002). Experimental insects were adults of both sexes, which had been blood-fed 7-14 days previously. As the bed bugs were normally fed weekly, this would have allowed enough time for the bugs to digest their blood meal but not to become starved (Mellanby, 1939). Starved bugs are bed bugs that have not been fed for a period greater than they have become accustomed to, in this case greater than one week. Recently engorged and starved bed bugs exhibit a low level of activity in the olfactometer (personal observation). Twelve hours before use, experimental insects were transferred into a pot and moved into the behaviour room to acclimatise.

\section{Olfactometer}

The still-air olfactometer consisted of a simple two-choice arena made from a Petri-dish lid (fig. 1) (Dawson et al., 1987) adapted to make the olfactometer suitable for bed bugs. Originally, the arena had no top, but to prevent escapes and to stop human odours contaminating the test environment, a Petri-dish base was used. On the floor of the arena were two holes (diameter, $26 \mathrm{~mm}$ ), $64 \mathrm{~mm}$ apart, under which pots $(60 \times 40 \mathrm{~mm})$ were placed. The arena was lined with fine muslin (pore size, $200 \mu \mathrm{m}$ ) to provide a suitable substrate for the bed bugs to walk on and to prevent contact with the volatile source. Two pots were present, a pot containing the bed bug-derived volatile source on filter paper $(70 \times 40 \mathrm{~mm})$ and a pot that contained clean filter paper $(70 \times 40 \mathrm{~mm})$, for convenience called the odour and the no-odour pot, respectively. The olfactometer was positioned inside a box screen $(28 \times 28 \times 23 \mathrm{~cm})$ as a precaution against directional bias that could be caused by variation within the room in environmental variables such as light and temperature. Clean cotton gloves were worn when handling equipment. After each replicate, olfactometers were washed with warm soapy water (Teepol) followed by $70 \%$ ethanol and air-dried before being used again.

\section{Experimental protocol}

Experiments were carried out in a controlled environment where the conditions were maintained at $25^{\circ} \mathrm{C} \pm 1.5^{\circ} \mathrm{C}$ and $80 \% \mathrm{RH} \pm 5 \%$. The light regime was set to $14 \mathrm{~L}: 10 \mathrm{D}$. As bed bugs are nocturnal, all bioassays were completed during the scotophase of the insect and red lamps (Jessops Darkroom Safelights) were used to facilitate observation. Before use, the red lamps were tested using a two-choice arena. The $>600 \mathrm{~nm}$ wavelength of light produced by the lamps was not found to have a significant photonegative effect on bed bug behaviour $\left(\chi^{2}=0.67, \mathrm{df}=1, P=0.41\right)$. Twenty replicates were completed in the presence and absence (control) of the volatiles using equal numbers of each sex. The odour pot was randomly assigned to one of the two positions, and the arena number, sex of the bed bug and order of treatments were also randomised. Controls were completed with two no-odour pots to test for bias. However, one pot was randomly assigned to be the odour pot for data analysis. Five minutes after setting up the equipment, the lid of the olfactometer was removed, and a bed bug was introduced into the centre of the arena using a paintbrush. This method was used as it was desirable to remove the bed bug from its refuge so that it would then exhibit refuge-seeking behaviour. The physical effects on the bed bug of using a paintbrush were found to be minimal and had no effect on its subsequent response to volatiles. The lid was then replaced and the bed bug was observed for 15 minutes. The following data were recorded by direct observation: (i) the 
first pot visit (odour or no-odour), (ii) the frequency with which bed bugs visited the area above the pots and (iii) the time spent active. Bed bugs that failed to make a choice were considered to be non-responders.

\section{Bed bug-derived volatiles}

Volatiles which occur naturally in bed bug refuges were tested to determine the suitability of the olfactometer for studying behavioural responses to potential semiochemicals. The response of adult bed bugs to bed bug-exposed material and conspecific bed bugs was tested. Bed bug-exposed papers, which consisted of faeces, cuticular compounds and any additional material deposited through contact with bed bugs, were chosen as a volatile source, as such papers have been shown to be attractive in previous studies (Levinson \& Bar Ilan, 1971; Siljander et al., 2007, 2008). In addition, the response to adult bed bugs was tested, as aggregating bed bugs may themselves produce semiochemicals that are attractive to refuge-seeking bugs. The bed bug-exposed material was filter paper that had been exposed to 100 bed bugs for one month. All bed bugs, exuviae and eggs were taken off the paper. The paper was then placed in a new pot, which was covered with clean mesh. For bed bug volatiles, ten adult bed bugs (five males and five females) were placed in a pot containing clean filter paper. The pot was covered with mesh and left for one week to allow the bed bugs to acclimatise. During this time, faeces and any other material that would accumulate in the presence of bed bugs was deposited on the papers, although it would be present in a lower concentration than on the bed bugexposed papers. The pots were kept under the same conditions as the colony.

\section{Statistical analyses}

Response and first visit to a pot (odour or no-odour) were analysed by two-sample binomial tests. A visit was defined as a period of time spent in the area directly above a pot and one visit lasted from the time of entry to this area until exit. A success was defined as a first visit to the odour pot, whereas a failure was defined as first visit to the no-odour pot or no choice. The null hypothesis was that there was no significant difference in the probability of success in the presence and absence of the volatiles. All continuous data were tested for normality prior to parametric analysis and were found to be normally distributed. All means displayed are, therefore, arithmetric means. The difference in the frequency of visits to the pots was analysed using a paired $t$-test with the null hypothesis that there was no significant difference in the number of visits to the odour and no-odour pots. Time spent active was converted to a proportion of activity (prop_active) for the duration of the experiment (15 min). Volatile presence, sex and time of day were all considered as model factors. The time of day was separated into early (10:00-15:00) and late scotophase (15:00-20:00). As the data contained missing values, which made the final design unbalanced, a Restricted Maximum Likelihood (REML) analysis was used. The proportions were analysed with the null hypothesis that there were no significant differences between the proportion of time spent active in the presence and absence of volatiles. All results were analysed using GenStat version 11.0 (Payne et al., 2008).
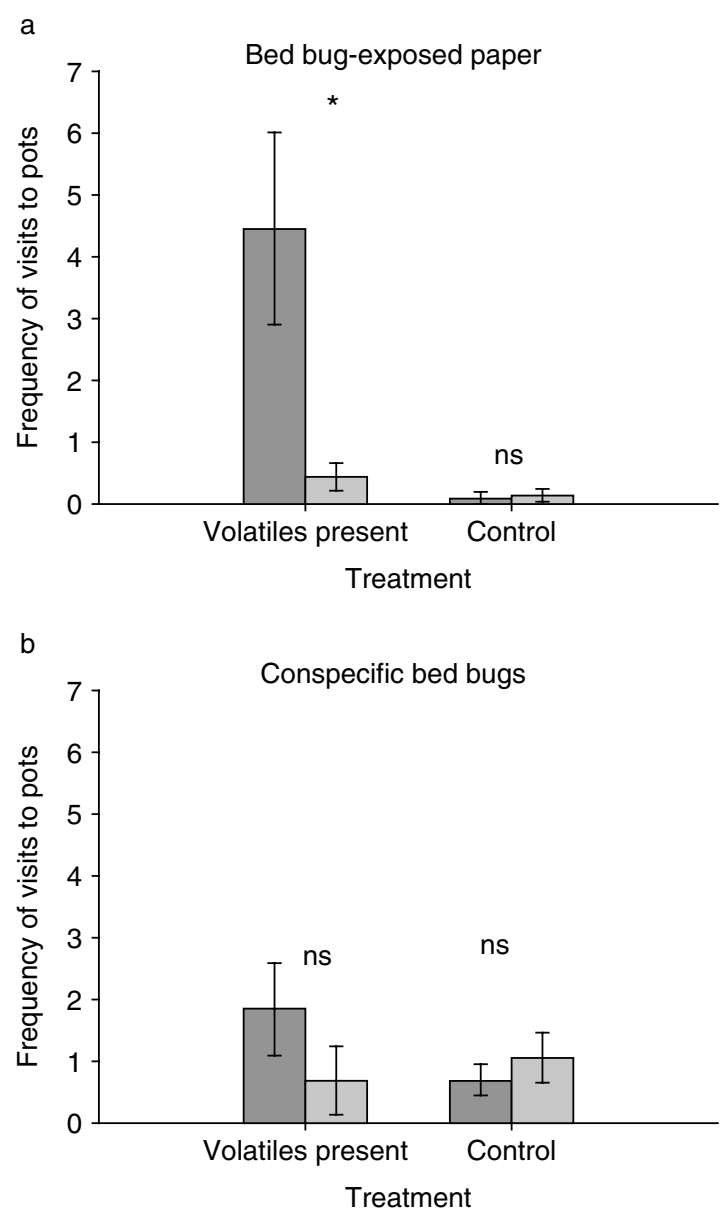

Fig. 2. The difference in mean frequency of visits to the area above the pots (odour-no-odour) by Cimex lectularius in the stillair Petri-dish olfactometer in the presence and absence (control) of volatiles from (a) bed bug-exposed paper and (b) bed bugs. Means \pm standard errors, asterix indicates significant differences in frequency of visits to the odour and no-odour pot ${ }^{*} P<0.05$, ns $P>0.05$. $\square$, odour pot; $\square$, no-odour pot.

\section{Results}

First choice

There was a significantly higher proportion of responsive bed bugs in the presence of volatiles from bed bug-exposed paper $(50 \%, n=20, P=0.006,95 \% \mathrm{CI}=0.1445,0.6555)$ compared with the response in the absence of the volatiles $(10 \%)$. Significantly more bed bugs chose to visit the odour pot first in the presence $(90 \%, n=20, P<0.001,95 \% \mathrm{CI}=0.2320$, $0.6680)$ of the volatiles compared with when the volatiles were absent $(0 \%)$.

There were no significant differences in the proportion of responsive bed bugs $(n=20, P=0.337,95 \% \mathrm{CI}=-0.4528$, $0.1528)$ in the presence (50\%) and absence (35\%) of volatiles from bed bugs. Additionally, there were no significant differences in the pot visited first in the presence $(70 \%)$ and absence $(90 \%)$ of the volatiles $(n=20, \quad P=0.519,95 \%$ $\mathrm{CI}=-0.4021,0.2021)$. 

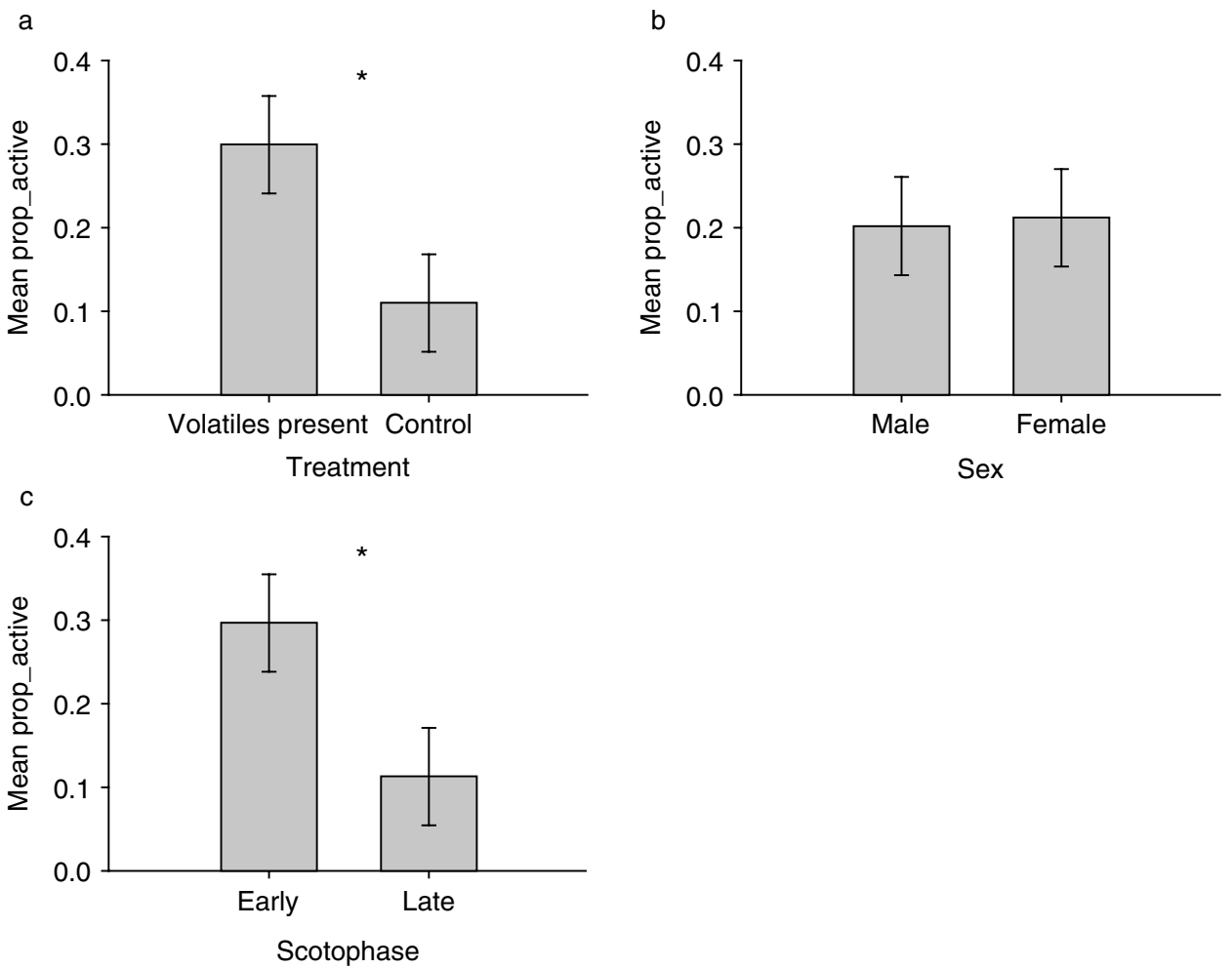

Fig. 3. Mean proportion of time spent active (prop_active) by Cimex lectularius in the still-air Petri-dish olfactometer (a) in the presence and absence (control) of volatiles from bed bug-exposed paper, (b) by sex and (c) at different time periods during the scotophase (early 10:00-15:00 and late 15:00-20:00). Means \pm standard errors; asterix indicates significant differences in prop_active ${ }^{*} P<0.05$.

\section{Frequency of visits}

Bed bugs were less likely to leave the sides of the arena and visit the area above the pots in the absence of the volatiles. In the presence of volatiles from bed bug-exposed papers, significantly more visits were made to the odour pot than the no-odour pot (fig. 2a; $t=2.83, \mathrm{df}=19, P=0.011$ ). During the control experiments, bed bugs showed no preference for either pot $(t=-0.44, \mathrm{df}=19, P=0.666)$.

In the presence of volatiles from bed bugs, the frequency with which bed bugs visited the two pots was not significantly different (fig. $2 \mathrm{~b} ; t=1.64, \mathrm{df}=19, P=0.117$ ). During control experiments, bed bugs showed no preference for either pot $(t=1.25, \mathrm{df}=19, P=0.226)$.

\section{Proportion of time spent active}

There was a significant difference in the proportion of time spent active (prop_active or activity) in the presence of volatiles from bed bug-exposed papers (fig. $3 a ; F_{1,32}=5.25$, $P=0.029)$. The sex of the bed bug had no significant effect on activity (fig. $3 b ; F_{1,32}=0.01, P=0.904$ ). The time of day at which the bioassays were completed (early or late scotophase) had a significant effect on the activity of bed bugs (fig. 3c; $F_{1,32}=4.97, P=0.033$ ). Bed bugs showed an increased level of activity independent of the presence of the volatiles and sex in the early scotophase (10:00-15:00). None of the interactions between treatment, sex and time were found to be significant (table 1).
Table 1. Statistical analysis of proportion of time spent active (prop_active) by Cimex lectularius in a still-air Petri-dish olfactometer. A table of interactions considering treatment (trt), sex and time of day as factors.

\begin{tabular}{lcccccc}
\hline & \multicolumn{7}{c}{ Proportion of time spent active (prop_active) } \\
\cline { 2 - 7 } & $\begin{array}{l}\text { Wald } \\
\text { Statistic }\end{array}$ & ndf & $\mathrm{F}_{\text {obs }}$ & ddf & $P$ & Significance \\
\hline \multicolumn{5}{c}{ Bed } & bug-exposed & papers \\
Trt.Time & 0.59 & 1 & 0.59 & 32.0 & 0.448 & $\mathrm{~ns}$ \\
Trt.Sex & 0.00 & 1 & 0.00 & 32.0 & 0.973 & $\mathrm{~ns}$ \\
Time.Sex & 0.31 & 1 & 0.31 & 32.0 & 0.584 & $\mathrm{~ns}$ \\
Trt.Time.Sex & 1.12 & 1 & 1.12 & 32.0 & 0.298 & $\mathrm{~ns}$ \\
& 0.32 & 1 & 0.32 & 14.3 & 0.583 & $\mathrm{~ns}$ \\
Trt.Time & 0.17 & 1 & 0.17 & 20.9 & 0.687 & $\mathrm{~ns}$ \\
Trt.Sex & 0.60 & 1 & 0.60 & 26.9 & 0.444 & $\mathrm{~ns}$ \\
Time.Sex & 1.00 & 1 & 1.00 & 19.9 & 0.330 & $\mathrm{~ns}$ \\
Trt.Time.Sex & \multicolumn{7}{c}{ Bed bugs } \\
\hline
\end{tabular}

ns, not significant when tested at the 0.05 level by REML analysis; ndf, numerator degrees of freedom; ddf, denominator degrees of freedom.

In the presence of volatiles from bed bugs, there was no significant difference found in the proportion of time spent active (fig. $4 \mathrm{a} ; \mathrm{F}_{1,14.1}=0.99, P=0.337$ ). There was a significant difference in activity dependent on the sex of the bed bug (fig. $4 \mathrm{~b} ; \mathrm{F}_{1,24.4}=8.18, P=0.009$ ). Female bed bugs were 

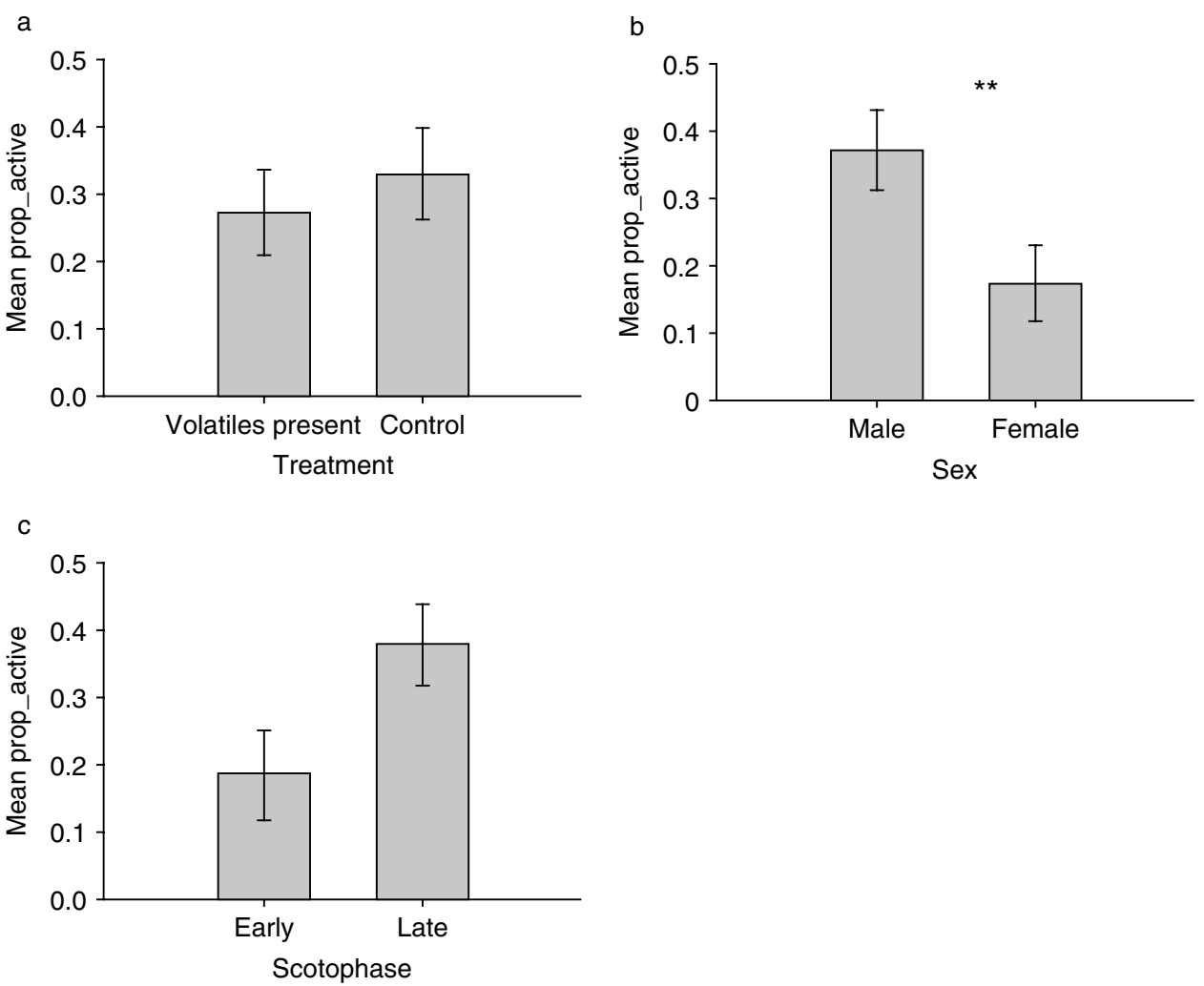

Fig. 4. Mean proportion of time spent active (prop_active) by Cimex lectularius in the still-air Petri-dish olfactometer (a) in the presence and absence (control) of volatiles from bed bugs, (b) by sex and (c) at different time periods during the scotophase (early 10:00-15:00 and late 15:00-20:00). Means \pm standard errors; asterix indicate significant differences in prop_active ${ }^{* *} P<0.01$.

significantly less active than male bed bugs independent of treatment and time. There was no significant effect of the time of day (early or late scotophase) on the activity of bed bugs during the bioassays (fig $4 \mathrm{c} ; \mathrm{F}_{1,16.5}=3.04, P=0.1$ ). None of the interactions between treatment, sex and time were found to be significant (table 1).

\section{Discussion}

\section{Response of $\mathrm{C}$. lectularius to bed bug-derived volatiles in a still-air olfactometer}

The present study modified a still-air olfactometer (Dawson et al., 1987) and successfully observed the responses of bed bugs to semiochemicals. Olfactory responses in the bed bug have been characterised by activation of the bed bug from its resting state, followed by antennal grooming, before random movement and lastly orientation towards the odour source (Aboul-Nasr \& Erakey, 1968b). In the olfactometer, bed bugs exhibited a wide range of behaviour, including walking, antennal grooming and orientation towards a volatile source.

Unlike olfactometers used in previous studies, the still-air Petri dish olfactometer separated bed bugs from any potential contact stimuli, enabling the study of chemotactic responses to volatiles without interference from thigmotaxis (Levinson \& Bar Ilan, 1971; Levinson et al., 1974a; Parashar et al., 2003; Siljander et al., 2007, 2008; Olson et al., 2009). The experimental protocol also involved the observation of the bed bugs over a short time frame, allowing the first choice and initial responses to the volatiles to be recorded. Previous studies were conducted over extended periods of time and only recorded the final choice (Levinson \& Bar Ilan, 1971; Levinson et al., 1974a; Parashar et al., 2003; Siljander et al., 2007, 2008; Olson et al., 2009). Studying initial responses to the volatiles provides a greater understanding of the behavioural role of the semiochemicals and will become much more relevant when designing a monitoring device.

The presence of volatiles from the bed bug-exposed paper caused a significant increase in bed bug activity and orientation towards the volatile source. Attraction of bed bugs to bed bug-exposed papers has been shown in previous studies, but this is the first time initial responses to these volatiles, in the form of attraction and increased activity, have been observed over a short time period (Levinson \& Bar Ilan, 1971; Siljander et al., 2007, 2008). Bed bugs could be responding to a pheromone, volatiles from faeces, cuticular compounds or a combination of these factors. Faeces and cuticular compounds would be at the highest concentration within the refuge and could, therefore, act as a guide or aggregation pheromone to refuge-seeking bed bugs. Aggregation semiochemicals in the refuge would indicate a suitable habitat with potential mates and the benefits that come with aggregating with conspecifics (Sinha \& Wallace, 1966; Turchin \& Kareiva, 1989; Benoit et al., 2007; Siljander et al., 2008). Release of aggregation semiochemicals often involves a high physiological investment due to the building of specialised glands and the production of vast quantities of 
pheromone (Cruz-Lopez et al., 2001; Wertheim et al., 2005). Production of semiochemicals via faeces is potentially a low investment way of producing a pheromone (Peng \& Weiss, 1992). As bed bug-exposed papers comprised material deposited by conspecific bed bugs, it is possible that aggregating bed bugs could produce semiochemicals that attract refuge-seeking bugs. However, when bed bugs were tested with volatiles from live bed bugs, there was no significant difference in activity and no significant orientation towards the volatile source. Previous studies have also recorded a lack of attraction to live bed bugs but attraction to bed bug refuges (Marx, 1955). It is possible that, in order for bed bug volatiles to be attractive, they may need to be presented in combination with refuge volatiles. Bed bug volatiles, especially if from a low number of individuals, may not be an indication of an established refuge and so may not be of interest to a refuge-seeking bug. Alternatively, if the bed bugs within the odour pot were stressed, they could produce an alarm pheromone and this would affect the behavioural response of the test insect (Levinson et al., 1974a). Whilst stress to all insects was minimised by allowing acclimatisation to conditions and handling pots and insects with care, it is very easy to cause release of this pheromone with unnatural stimulation or changes in conditions (Reinhardt \& Silva-Jothy, 2007).

\section{Effect of photoperiod}

There was a significant increase in activity in the early scotophase independent of the presence of volatiles. Bed bugs are nocturnal insects that aggregate diurnally. Therefore, it may be expected that bed bugs would disperse as light intensity decreases and be at their most aggregated by the end of the scotophase. A study performed in a semi-field situation, an animal house, found that bed bugs were at their most active between 03:00 and 06:00 (Mellanby, 1939). It is possible that, during the current study, a second peak of activity was present at the end of the scotophase, which would have escaped detection as bioassays were not completed in the last three hours of the scotophase. In addition, differences between the two studies may be associated with host behaviour. The animal house held mainly rats, and bed bugs may have adjusted their feeding behaviour to feed on these nocturnal mammals during a time when the insectary was not used and the rats were inactive.

\section{Effect of sex}

In this experiment, females were less active than males regardless of treatment or time. Physiological effects are more pronounced in female bed bugs due to the energy demands of reproduction. Although both sexes (and all nymphal instars) are obligate blood feeders, females take bigger blood meals and need to feed more frequently than males and nymphs (Jones, 1930). The rate of metabolism in a reproductively active female was found to be equal to that of a male that was kept moving continuously (Mellanby, 1939). Females may be generally less active than males during the bioassays, as it is important for them to conserve energy for feeding and oviposition.

\section{Potential of bioassay}

An aggregation pheromone, which is yet to be identified, could be exploited to develop effective monitoring tools to detect and control bed bug infestations. In order to identify semiochemicals associated with bed bug aggregation, knowledge of bed bug biology and behaviour is required. The current study has identified a suitable olfactometer and shown attraction to bed bug-derived volatiles from bed bugexposed papers, but not to live bed bugs. The study has also highlighted differences in bed bug activity dependent upon the sex of the test insect and the time of day that the bioassays were completed. These differences need to be investigated further and should be considered when designing experiments in the future. This study has provided a basis for investigations into bed bug chemical ecology, including the role and identification of potential semiochemicals for monitoring or control of this important medical and veterinary insect pest.

\section{Acknowledgements}

E.N.I. Weeks was supported by a BBSRC CASE Studentship with AgriSense BCS Ltd. Rothamsted Research receives grant-aided support from the BBSRC. We would like to thank Lynda Castle for assistance with the olfactometer diagram. Cimex lectularius were donated by Dr Nigel Hill, London School of Hygiene and Tropical Medicine.

\section{References}

Aboul-Nasr, A.E. \& Erakey, M.A.S. (1968a) The effect of contact and gravity reactions upon the bed-bug, Cimex lectularius L. Bulletin Societe Entomologue d'Egypte 52, 363-370.

Aboul-Nasr, A.E. \& Erakey, M.A.S. (1968b) Behaviour and sensory physiology of the bed-bug, Cimex lectularius L., to some environmental factors: chemoreception (Hemiptera: Cimicidae). Bulletin Societe Entomologue d'Egypte 52, 353362.

Benoit, J.B., Del Grosso, N.A., Yoder, J.A. \& Denlinger, D.L. (2007) Resistance to dehydration between bouts of blood feeding in the bed bug, Cimex lectularius, is enhanced by water conservation, aggregation, and quiescence. American Journal of Tropical Medicine and Hygiene 76, 987-993.

Boase, C. (2004) Bed-bugs - reclaiming our cities. Biologist 51, 102-103.

Cruz-Lopez, L., Malo, E.A., Rojas, J.C. \& Morgan, E.D. (2001) Chemical ecology of triatomine bugs: vectors of Chagas disease. Medical and Veterinary Entomology 15, 351-357.

Dawson, G., Griffiths, D., Janes, N., Mudd, A., Pickett, J., Wadhams, L. \& Woodcock, C. (1987) Identification of an aphid sex pheromone. Nature 325, 614-616.

Doggett, S., Geary, M. \& Russell, R. (2004) The resurgence of bed bugs in Australia: With notes on their ecology and control. Environmental Health 4, 30-38.

Harlan, H. (2006a) Technical guide 44: bed bugs - importance, biology, and control strategies. 15 pp. Washington, DC, USA, Defense Pest Management Information Analysis Center, Armed Forces Pest Management Board.

Harlan, H.J. (2006b) Bed Bugs 101: the Basics of Cimex lectularius. American Entomologist 52, 99-101.

Harlan, H. (2007) Bedbug control: Challenging and still evolving. Outlooks on Pest Management 18, 57-61.

Harlan, H., Faulde, M. \& Baumann, G. (2008) Bed bugs. pp. 131-154 in Bonnefoy, X., Kampen, H. \& Sweeney, K. (Eds) Public Health Significance of Urban Pests. Copenhagen, Denmark, World Health Organisation Europe. 
Jones, R.M. (1930) Some effects of temperature and humidity as factors in the biology of the bedbug (Cimex lectularius Linn.). Annals of the Entomological Society of America 23, 105-119.

Levinson, H.Z. \& Bar Ilan, A.R. (1971) Assembling and alerting scents produced by the bedbug Cimex lectularius L. Experientia 27, 102-103.

Levinson, H.Z., Levinson, A.R. \& Maschwitz, U. (1974a) Action and composition of alarm pheromone of bedbug Cimex lectularius L. Naturwissenschaften 61, 684-685.

Levinson, H.Z., Levinson, A.R., Muller, B. \& Steinbrecht, R.A. (1974b) Structure of sensilla, olfactory perception, and behaviour of bedbug, Cimex lectularius, in response to its alarm pheromone. Journal of Insect Physiology 20, 1231-1248.

Marx, R. (1955) Uber die wirtsfidung und die bedeutung des artspezifischen duftstoffes bei Cimex lectularius Linne. Zeitschrift fur Parasitenkunde 17, 41-72.

Mellanby, K. (1939) The physiology and activity of the bed-bug (Cimex lectularius) in a natural infestation. Parasitology 31, 200-211.

Montes, C., Cuadrillero, C. \& Vilella, D. (2002) Maintenance of a laboratory colony of Cimex lectularius (Hemiptera: Cimicidae) using an artificial feeding technique. Journal of Medical Entomology 39, 675-679.

Moore, D.J. \& Miller, D.M. (2009) Field evaluations of insecticide treatment regimens for control of the common bed bug, Cimex lectularius (L.). Pest Management Science 65, 332-338.

Myamba, J., Maxwell, C., Asidi, A. \& Curtis, C. (2002) Pyrethroid resistance in tropical bedbugs, Cimex hemipterus, associated with use of treated bednets. Medical and Veterinary Entomology 16, 448-451.

Olson, J.F., Moon, R.D. \& Kells, S.A. (2009) Off-host aggregation behavior and sensory basis of arrestment by Cimex lectularius (Heteroptera: Cimicidae). Journal of Insect Physiology 55, 580-587.

Parashar, B.D., Ganesan, K., Sukumaran, D., Rao, Y.V.S., Veer, V. \& Prakash, S. (2003) Aggregation activity induced by excreta extracts in Cimex hemipterus (Hemiptera: Cimicidae). Entomon 28, 215-222.

Payne, R.W., Murray, D.A., Harding, S.A., Baird, D.B. \& Soutar, D. (2008) GenStat for Windows (11th Edition) Introduction. In. VSN International, Hemel Hempstead.
Peng, C.W. \& Weiss, M.J. (1992) Evidence of an aggregation pheromone in the flea beetle, Phyllotreta crucifereta (Goeze) (Coleoptera, Chrysomelidae). Journal of Chemical Ecology 18, 875-884.

Potter, M.F. (2005) A bed bug state of mind: emerging issues in bed bug management. Pest Control Technology 33, 82-85.

Potter, M.F., Romero, A. \& Haynes, K.F. (2008) Battling bed bugs in the USA. pp. 401-406 in Proceedings of the 6th International Conference on Urban Pests. Robinson, W.H. \& Bajomi, D. (Eds) Congress Center, Budapest, 13-16 July 2008, Executive Committee of the International Conference on Urban Pests.

Reinhardt, K. \& Silva-Jothy, M.T. (2007) Biology of the bed bugs (Cimicidae). Annual Review of Entomology 52, 351-374.

Richards, L., Boase, C., Gezan, S. \& Cameron, M. (2009) Are bed bug infestations on the increase within Greater London? Journal of Environmental Health Research 9, 17-24.

Romero, A., Potter, M.F., Potter, D.A. \& Haynes, K.F. (2007) Insecticide resistance in the bed bug: A factor in the pest's sudden resurgence? Journal of Medical Entomology 44, 175178.

Siljander, E., Penman, D., Harlan, H. \& Gries, G. (2007) Evidence for male- and juvenile-specific contact pheromones of the common bed bug Cimex lectularius. Entomologia Experimentalis et Applicata 125, 215-219.

Siljander, E., Gries, R., Khaskin, G. \& Gries, G. (2008) Identification of the airborne aggregation pheromone of the common bed bug, Cimex lectularius. Journal of Chemical Ecology 34, 708-718.

Sinha, R.N. \& Wallace, H.A.H. (1966) Ecology of insect-induced hot spots in stored grain in western Canada. Research in Population Ecology 8, 107-132.

Turchin, P. \& Kareiva, P. (1989) Aggregation in Aphis varians an effective strategy for reducing predation risk. Ecology 70, 1008-1016.

Weerakone, I. (2007) Two aspects of the use of insecticidal treated bednets in Tanzanian villages. PhD thesis, London School of Hygiene and Tropical Medicine, London, UK.

Wertheim, B., Van-Baalen, E., Dicke, M. \& Vet, L. (2005) Pheromone-mediated aggregation in non-social arthropods: An evolutionary perspective. Annual Review of Entomology 50, 321-346. 